solution to a small cut or scarification in the skin produces an urticarial lesion quite similar in appearance to the reactions of individuals hypersensitive to pollen, dandruff and feather extracts. Sollman ${ }^{1}$ has reported observations on the effect upon the skin reactivity of repeatedly applying histamine to the same site. In agreement with his results, we have found that the histamine skin reaction is not exhaustible-on the contrary, it progressively increases with each subsequent application on the same area. Since histamine is non-antigenic, the non-specific skin reaction produced by it can not be dependent upon an antigen-antibody reaction. To us, it seems significant that this non-antigenic substance produces a local reaction which is not exhaustible, while the antigenic substances which we have used in the skin reactions on hypersensitive patients produce a reaction which may easily be completely exhausted.

Since our observations demonstrate that it is possible to abolish locally the reactivity of the skin, it seemed possible that by local application of pollen or dandruff extracts to the mucous membrane of the nose and throat of patients with allergic rhinitis, the reactivity might here also be abolished. We have not as yet carried far enough this therapeutic application of our observations to draw conclusions, but the results, so far as they go, are very satisfactory.

109 (1691)

\title{
Preliminary report on a staphylococcus bacteriophage.
}

BY ATDRÉ GRATIA.

[From the Laboratories of the Rockefeller Institute for Medical Research.]

In 1915, two years before the discovery of d'Hérelle, Twort described the following phenomenon. ${ }^{2}$ If glycerinated calf vaccinia is streaked on agar slants, a certain number of the micrococcus colonies which grow become glassy and transparent, and degenerate into a granular material which cannot be subcultured and which, even when diluted $I, 000,000$ times and filtered, gives rise to the same degeneration when added to a normal culture of micrococci, and so on indefinitely.

'Sollmann, T., and Pilcher, J. D., Jour. Pharm. and Exp. Ther., 1919, ix, 309.

2 The Lancet, 1915, ii, I24I. 
Because of the similarity between these observations and the phenomenon of d'Herelle, the following experiments were undertaken with the hope of obtaining from vaccinia a typical bacteriophage for staphylococci.

Several agar slants were seeded with untreated fresh green vaccinia pulp. The growth, consisting of Staphylococcus albus, Staphylococcus aureus, and B. coli, looked normal in all tubes but one, in which a few small, clarified areas were found.

Filtrates obtained from subcultures of these clarified spots were found to possess a marked inhibiting and dissolving action on the growth of staphylococci, and this lysis could be carried on indefinitely from one culture to another.

Staphylococcus is, therefore, the first Gram-positive bacterium for which an observation of transmissible autolysis has been made. Attempt to extend the lysis to other cocci has thus far been unsuccessful.

As has already been observed for other species, great variations in sensitiveness exist not only among different strains of staphylococci-certain ones remaining unaffected-but also between different organisms of a single strain, a few individuals usually being able to resist solution.

If a partially dissolved culture of staphylococcus is streaked on agar plates, it is found that in the first streak, where the lytic broth is spread abundantly together with the cocci which are still alive, the growth is poor, irregular and glassy. In the subsequent streaks, the colonies are less irregular, and finally become normally round and opaque. A round colony, transplanted, gives only round colonies, while an irregular colony gives colonies which, irregular in the first streaks, become less irregular and finally round in the following streaks.

In agreement with the observations described by Kuttner ${ }^{1}$ for typhoid bacilli, and with our own observations on B. coli, an irregular colony was found to be lysogenic, a regular colony nonlysogenic.

In the course of our previous studies on coagulation of blood we observed a coagulant effect exerted by staphylococcus on all kinds of non-spontaneously-coagulable blood plasma (oxalated,

1 Proc. Soc. EXPEr. Brol. AND MEd., 1921, xviii, 158. 
citrated, salted, hirudinized, filtered plasma, etc.). ${ }^{1}$ We have now observed that sterile filtrates of the above mentioned dissolved cultures of staphylococci possess the same property.

$$
\text { I10 (1692) }
$$

On the preparation of a soluble protein extract from soy beans.

By SELMAN A. WAKSMAN.

[From the New Jersey Agricultural Experiment Station, New Brunswick, N. J.]

The average protein content of the soy bean is 36.5 per cent. while that of the soy bean cake is about $4 \mathrm{I}$ per cent. The protein of the soy bean has all the amino acids necessary for nutrition and it is claimed to be as valuable as the casein of milk. The proteins of the soy bean, unlike those of other leguminous plants thus far investigated, were found, by Osborne and Mendel, to be adequate for promoting normal growth. In addition to the high protein content, the soy bean is found to contain an adequate amount of water-soluble vitamine and some essential fat-soluble vitamine. These factors combined with low cost make the soy bean of unique significance as a food.

In working with proteolytic enzymes of fungi, the author found some organisms that are able to develop active enzymes which readily hydrolize the soy bean proteins and make them soluble. By using the ground soy bean cake as a substratum for the growth of the proteolytic fungus, then, at the end of the proper incubation period, which is usually about 36 hours, adding water to the mass and allowing the enzyme present in the mycelium to act upon the soy bean proteins, over 50 per cent of the proteins are found to go into solution. This solution when concentrated gives a material containing 45 or more per cent of soluble proteins and protein degradation products and is quite rich in vitamines. This extract can be very cheaply prepared and it may take the place, when properly modified by the addition of necessary salts, of meat extract and other digested meats in infant feeding and, since the

1 Compt. rend. Soc. Biol., I919, 1xxxii, I245, I247, 1393; I920, 1xxxiii, 649, 584, 585,649 . 